\title{
Unusual implant-related soft tissue reaction presenting as a swollen leg: a case report
}

\author{
Seyma Ozkanli ${ }^{1}$, Mehmet Salih Soylemez ${ }^{2, *^{*}}$, Adem Sahin ${ }^{2}$, Serkan Senol ${ }^{1}$, Kerem Bilsel ${ }^{3}$ and Hasan Hüseyin Ceylan ${ }^{3}$
}

\begin{abstract}
Introduction: There are several causes of peri-implant edema, pain, and swelling around implants after orthopedic fixation device application for fracture repair. The most common and well-known reason is infection, however, granulomas associated with foreign body reactions are rarely seen. In this report we present a case of a granulomatous reaction mimicking a hydatid cyst and sarcoma. We emphasize the importance of differential diagnosis in triggering appropriate management of the patient. Our case was unusual; to the best of our knowledge no similar clinical or pathological findings have been reported in current literature.
\end{abstract}

Case presentation: A 56-year-old Turkish man who had been treated for a right femoral fracture via a plate fixation 10 years prior underwent an operation to treat pain and swelling around the plate. A hydatid cyst-like mass was observed during surgery, but subsequent examination revealed that no hydatid cyst was present; both malignancy and infection were also absent.

Conclusions: Although infection is generally the first possibility that should be considered in a patient complaining of pain and swelling in the vicinity of an implant, malignancies, hydatid cysts, and (finally) foreign body reactions should also be kept in mind as differential diagnoses. A soft-tissue reaction around a previously implanted plate should be managed carefully. Pre-operative radiological assessment, and biopsy to allow pathological and microbiological examination, should be considered in all suspected cases.

Keywords: Soft-tissue reaction, Granuloma, Malignancy, Orthopedic hardware

\section{Introduction}

There can be several causes of peri-implant edema, pain, and swelling around the implant after orthopedic fixation device application for fracture repair. The most common and well-known reason is infection however, granulomas associated with foreign body reactions are rare. Swelling and pain may develop around the implant in patients with sarcomas. Staphylococcus spp. are the most common infectious organisms associated with orthopedic implant surgery. Furthermore, hydatid cysts, especially in patients with predisposing factors, may cause implant infections [1]. Echinococcus granulosus is usually responsible for the more commonly encountered hydatid diseases and/or infections [2]. Although uncommon, the development of a

\footnotetext{
* Correspondence: slhsylmz@gmail.com

${ }^{2}$ Department of Orthopedics and Traumatology, Göztepe Training and Research Hospital, Fahrettin Kerim Gökay Street, 2346 Istanbul, Turkey ${ }^{4}$ Göztepe E.A.H. floor 3. Orthopaedics ward, Fahrettin Kerim Gökay Street, 2346 Kadıköy/Istanbul, Turkey

Full list of author information is available at the end of the article
}

sarcoma due to the presence of an orthopedic implant can be caused by swelling after the implantation. To the best of our knowledge, thirty-one cases of sarcoma development due to an orthopedic implant have been reported in current literature [3].

Sarcoma development due to implants can take several forms, but the most common are malignant fibrous histiocytoma and osteosarcoma. In addition, there has been one report of a peripheral nerve-sheath tumor and two reports of lymphoma resulting from implants [3]. These types of tumoral mass behave aggressively and frequently metastasize. Clinically, these cases should be distinguished from non-neoplastic reactions, such as infection, and reactions to the prosthetic debris associated with implants. Our case report involves a patient who had been treated for a right femoral fracture by fixation with a plate. Ten years later the patient underwent an operation due to pain and swelling around the plate. During surgery, a hydatid cyst-like mass was observed, but subsequent examination revealed no malignancy, infection, or hydatid cyst. 


\section{Case presentation}

A 56-year-old Turkish man with no history of illness or drug use was treated with plate and screw osteosynthesis because of a right femoral fracture after a vehicle accident in 1998. He had been living with and feeding a dog for the past 16 years. Two years prior to the current presentation, he noticed a slight swelling on the proximal lateral aspect of his right thigh, but he did not pursue evaluation. However, the symptoms had been increasing during the most recent six months, and he was admitted to our clinic. His C-reactive protein (CRP) level was $3.7 \mathrm{mg} / \mathrm{dl}$ and his eosinophil concentration was $12.4 \%$. A biopsy specimen was taken from the area of swelling because of suspected infection. The biopsy specimen was considered to involve greater trochanter bursitis. However, because of increasing swelling and pain during the three months following the biopsy, he was re-evaluated in our clinic. A $25 \times 12 \mathrm{~cm}$ mass was present on the proximal anterolateral aspect of the right thigh, starting at the greater trochanter and spreading both distally and posteriorly. There was no warmth or redness associated with the mass. A diagnosis of bursitis of the greater trochanter was made due to the swelling, which was particularly severe on the greater trochanter and the screw heads on the proximal greater trochanter. To identify the causative organism, the mass was punctured again. The culture grew methicillin-sensitive, coagulase-negative Staphylococcus (Staphylococcus epidermidis), which was determined to be due to contamination. Tests for a potential infection were taken. His test results showed a sedimentation rate of $107 \mathrm{~mm} / \mathrm{h}, \mathrm{CRP}$ of $1.38 \mathrm{mg} / \mathrm{dl}$, a white blood cell count of $7.8 \times 10^{3} / \mathrm{mm}^{3}$, and eosinophils of 2.4 . He was diagnosed with bursitis of the trochanter major, and removal of the bursitis and an implant operation were planned. The excised mass started from the lateral aspect of the distal femur (next to the implant) and spread postero-superiorly to the level of the greater trochanter, which was $15 \times 12 \times 6 \mathrm{~cm}$ in diameter. The mass was filled with a $1 \times 1 \mathrm{~cm}$ cystic structure that appeared to be comprised of hydatid cyst vesicles (Figures 1 and 2). During surgery, the mass was excised with preservation of the sciatic nerve as the cyst had burst. Bone tissue had not developed on the implant and the plates and screws were removed from the patient. Intra-operatively, we considered that the mass might have been a hydatid cyst or a sarcoma, and thus post-operatively, the patient underwent contrast-enhanced abdominal and thoracic computed tomography $(\mathrm{CT})$. His CT scan revealed neither a primary focus nor any other area of involvement. An abdominal ultrasonography examination was unremarkable. A direct parasitic examination of the specimens taken intraoperatively showed no protoscoleces within the cyst. Samples were taken intra-operatively and sent for pathological examination. The diagnosis was verified and the softtissue reaction evaluated. Twelve months after the procedure our patient was fully mobilized and determined to be in a good general condition. A follow-up CT scan of his abdomen, chest, and right thigh revealed no secondary focus.

\section{Discussion}

Although implants are biologically inactive materials, they may cause soft-tissue reactions [4]. The most common causes of peri-implant swelling and pain in the midto long-term period after orthopedic internal fixation device implantation are infections and soft-tissue reactions, caused by metallic debris originating from these materials. Staphylococcus spp. are the most common causative organisms of infections that develop after orthopedic implant surgery [1]. In a study by Arciola et al. [1], Staphylococcus spp. were detected in 43 out of 50 (86\%) orthopedic implants. Moreover, Staphylococcus aureus was the only detected microorganism in 34 patients (68\%). In our case, the pre-operative radiographs were normal, there were no signs of a loosening of the implant, and the bone plate attachment was quite firm. The intra-operative cultures were sterile. The large number of snowflake-like cysts, $1 \times 1 \mathrm{~cm}$ in size and demonstrating a cystic structure, were thought to be hydatid cysts as our patient was known to have a dog.

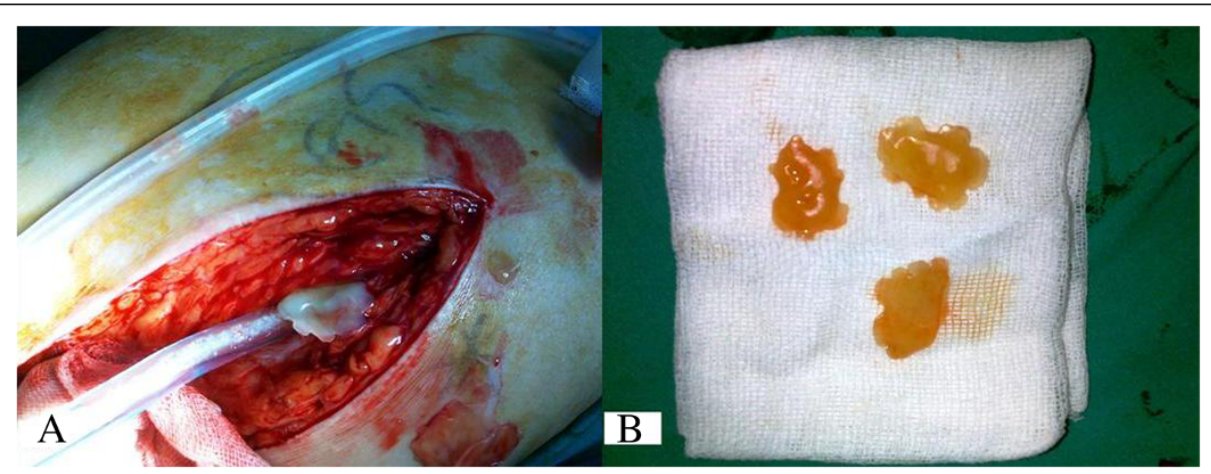

Figure 1 Image of snow-shaped vesicles extracted from the mass (A, B). 


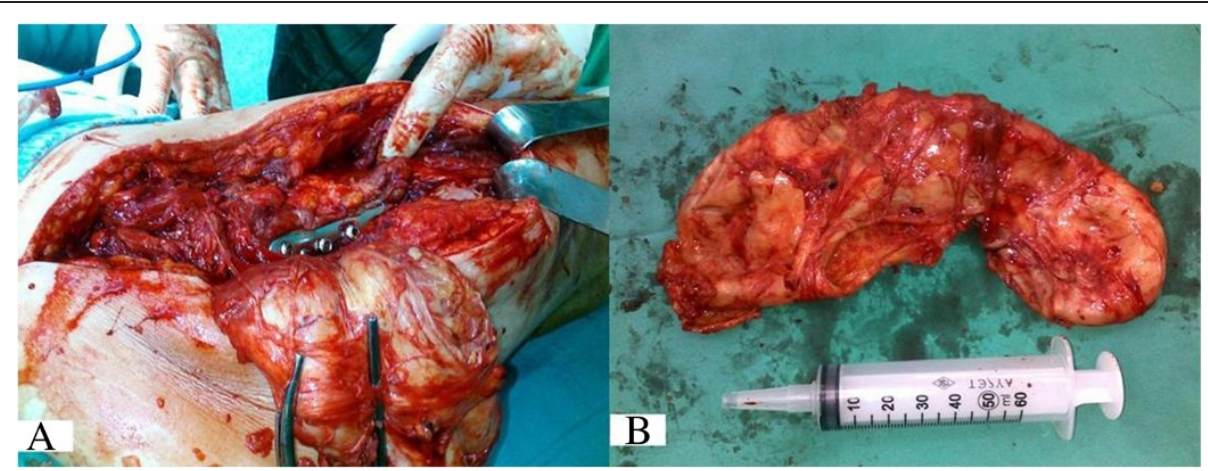

Figure 2 Image of the capsule, dissected from the stainless steel plate (A, B).

The hydatid cyst, which contained an outer fibrous macroscopic laminar layer and an inner germinal layer, had a so-called two-layered cyst wall containing a clear liquid. The germinal membrane contained small cysts and protoscoleces. Hydatid-related skin [5], brain [6], pancreas [7], leg, and spine [8] disease has been reported, but such a disease would not be expected to cause orthopedic implant infections. Therefore, local changes in the incision after a previous orthopedic surgery may direct the practitioner to investigate other bacterial pathogens, potentially leading to diagnostic errors. If a patient's laboratory results are atypical at the wound site of a non-specific infection following orthopedic surgery, and the patient has a history of travelling to a region endemic for hydatid disease as well as contact with dogs, a diagnosis of hydatid cyst disease should be considered [9]. However, in our patient, his post-operative tests revealed no evidence of hydatid disease. The intra-operative findings were indicative of hydatid disease but, upon pathological and parasitological examination, neither scolices nor protoscoleces were detected.

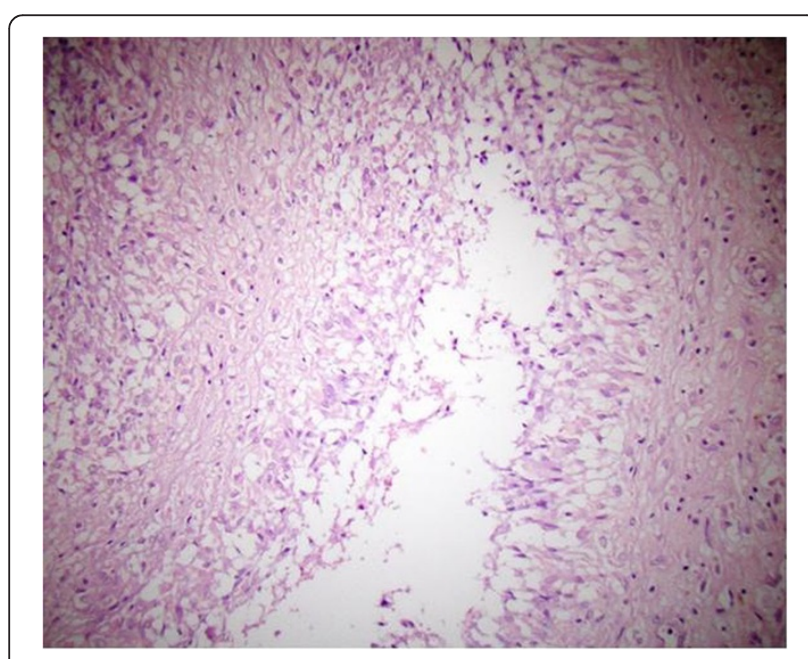

Figure 3 Lymphocytic and histiocytic inflammation in soft tissue. (Stain, hematoxylin and eosin; original magnification, $\times 100$ ).
The most serious pathology that might trigger periimplant edema and swelling after the use of orthopedic implants and prostheses is malignancy. Various animal studies have examined the biological effects of implants. Beryllium, cadmium, chromium, cobalt, iron, nickel, selenium, zinc, and titanium carcinogenicity studies have been conducted to demonstrate these effects [10-14]. However, some authors place the implant in accordance with the sarcoma found which can lead to the development of osteonecrosis [15]. A total of 31 cases of sarcoma associated with implants have been reported in the current literature. Such sarcomas may take various forms, the most common being malignant fibrous histiocytoma and osteosarcoma [3]. They behave aggressively and frequently metastasize. Sarcomas associated with implants may also develop in relation to the presence of metallosis and prosthetic debris. In 2001, Suzanne et al. [16] studied 12 patients who developed implant-associated sarcomas. Sarcomas were found in five patients after a total hip replacement, in four patients after the intramedullary nailing of fractures, in two patients after a staple

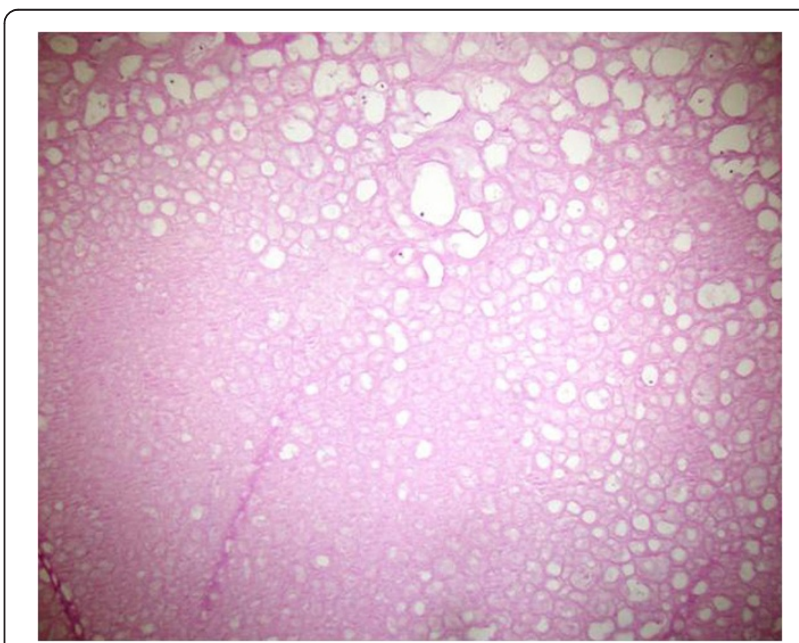

Figure 4 Acellular fibro-hyalinized structure. (Stain, hematoxylin and eosin; original magnification, $\times 40$ ). 
application for osteotomy fixation, and in one patient after a plate fixation for a femoral fracture [3]. A stainless steel plate had been used to treat our patient. Similar reactions may thus be expected in other patients treated with stainless steel implants. However, such reactions should be differentiated from malignancies.

In our case, there was no evidence of intra-operative metallosis. The dimensions of the removed mass approached $15 \times 12 \times 6 \mathrm{~cm}$. A pathologic examination revealed the cystic structures detected to have a wall thickness approaching $1 \mathrm{~mm}$. The largest of the cysts was $2 \mathrm{~cm}$ in diameter, with the smallest being $2 \mathrm{~mm}$ in diameter. The small cystic structures that were extruded from the mass were 3 to $4 \mathrm{~mm}$ thick, numerous, dirty white in color, smoothsurfaced, rubber-like, and flat. Paraffin sections were stained with hematoxylin and eosin, and acellular, fibro-hyalinized cystic structures were observed (Figures 3 and 4). No protoscoleces were detected. Evaluation of the surrounding soft tissue revealed lymphocytes, polymorphonuclear leukocytes, and inflammatory cell infiltrations comprising histiocytes. The slices exhibited minimal cell wall structural features, mitosis, and/or necrosis. Atypical cells were not considered to indicate malignancy because of their rarity.

Although infection is generally the first possibility that should be considered in patients with pain and swelling in the vicinity of a prior implant, malignancies, hydatid cysts, and (finally) foreign body reactions should be kept in mind as differential diagnoses. No detailed investigation, such as magnetic resonance imaging, had previously been performed in our patient. Punctures were performed twice before the existence of greater trochanter bursitis was assumed, but the specimens were not sent for histopathological examination. This constitutes improper management of our patient. Insufficient pre-operative workup in such a case can result in an erroneous diagnosis, such as a hydatid cyst or a sarcoma. Therefore, we had planned to perform a wide resection following a possible diagnosis of malignancy or a hydatid cyst.

\section{Conclusions}

In conclusion, the current literature does not report on any case exhibiting similar clinical and pathological findings to the best of our knowledge. A soft-tissue reaction around a previously implanted plate should be managed carefully. Our case is important because, despite the similarity between a macroscopic hydatid cyst and a sarcoma, they are only microscopically distinguishable from a soft-tissue reaction.

\section{Consent}

Written informed consent was obtained from the patient for publication of this manuscript and any accompanying images. A copy of the written consent is available for review by the Editor-in-Chief of this journal.

\section{Abbreviations}

CRP: C-reactive protein; CT: Computerized tomography.

\section{Competing interests}

The authors declare that they have no competing interests.

\section{Authors' contributions}

AS, KB, SS and HHC contributed to the conception and design of the study carried out the literature research, manuscript preparation and manuscript review. SO performed the histological examination of the mass, and was a contributor in writing the manuscript. MSS was involved with the case and writing of the manuscript, general management of the patient and revised the manuscript for important intellectual content. All authors read and approved the final manuscript.

\section{Author details}

${ }^{1}$ Department of Pathology, Göztepe Training and Research Hospital, Fahrettin Kerim Gökay Street, 2346 Istanbul, Turkey. ${ }^{2}$ Department of Orthopedics and Traumatology, Göztepe Training and Research Hospital, Fahrettin Kerim Gökay Street, 2346 Istanbul, Turkey. ${ }^{3}$ Department of Orthopedics and Traumatology, School of Medicine, Bezmialem Vakıf University, Vatan Street, 34080 Istanbul, Turkey. ${ }^{4}$ Göztepe E.A.H. floor 3. Orthopaedics ward, Fahrettin Kerim Gökay Street, 2346 Kadıköy/lstanbul, Turkey.

Received: 10 February 2014 Accepted: 31 March 2014

Published: 13 June 2014

\section{References}

1. Arciola CR, Cervellati M, Pirini V, Gamberini S, Moıntanaro L: Staphylococci in orthopedic surgical wounds. New Microbiol 2001, 24:365-9.

2. Bengisun U, Tunç G, Kesenci M, Kuzu I, Demirci S, Aliç B: Surgical treatment of advanced alveolar hydatid disease of the liver: a report of five cases. Surg Today 1997, 27:261-5.

3. Keel SB, Jaffe KA, Nielsen GP, Rosenberg AE: Orthopedic implant-related sarcoma: a study of twelve cases. Mod Pathol 2001, 12:969-977.

4. Jacobs JJ, Skipor AK, Black J, Urbam RM, Galante JO: Release and excretion of metal in patients who have a total hip replacement component made of titanium-base alloy. J Bone Joint Surg 1991, 73-A:1475-1486.

5. Bresson-Hadni S, Humbert P, Paintaud G, Auer H, Lenys D, Laurent R, Vuitton DA, Miguet JP: Skin localization of alveolar echinococcosis of the liver. J Am Acad Dermatol 1996, 34:873-7.

6. Ihara T, Imai T, Saito H, Tashiro K, Onmura Y: Cerebral alveolar hydatid cyst-case report. NeuroMed Chir (Tokyo) 1991, 31:342-5.

7. Frossard JL, Armenian B, Gottstein B, Diebold-Berger S, Khan H, Vine R, Hadengue A: A case of alveolar echinococcosis restricted to pancreas. Am J Gastroenterol 1997, 92:2117-9.

8. Kunze V, Layer G, Brunning R, Nagele M: Metastasizing echinococcus alveolar of the liver. Radiology 1992, 32:444-7.

9. Uysal V, Paksoy N: Echinococcosis multilocularis in Turkey. J Trop Med Hyg 1986, 89:249-255.

10. Health JC, Daniel MR: The production of malignant tumors by cadmium in the rat. Br J Cancer 1964, 18:124-129.

11. Health JC, Daniel MR: The production of malignant tumors by nickel in the rat. Br J Cancer 1964, 28:261-264.

12. Heath JC, Freeman MAR, Swanson SAV: Carcinogenic properties of wear particles from prostheses made in cobalt-chromium alloy. Lancet 1971, 1:564-566.

13. Sundermann FW: Metal carcinogenesis in experimental animals. Food Cosmet Toxical 1971, 9:1056-120.

14. Pedley EB, Meachim G, Williams DF: Tumor induction by implant materials. In Fundamental aspects of biocompatibility. Volume 2. Edited by Williams DF. Boca Raton: CRC Press; 1981:175.

15. Hamblen DL: Sarcoma and jont replacement (editorial). J Bone Jont Surg 1984, 66-B:625-627.

16. Suzanne BK, Kenneth AJ, Nielsen GP, Andrew ER: Orthopadic Implant-related sarcoma: a study of twelve cases. Mod Pathol 2001, 14(10):969-967.

\section{doi:10.1186/1752-1947-8-187}

Cite this article as: Ozkanli et al.: Unusual implant-related soft tissue reaction presenting as a swollen leg: a case report. Journal of Medical Case Reports 2014 8:187. 